\title{
Drainage Radius after High Pressure Water Jet Slotting Based on Methane Flow Field
}

\author{
Binwei Xia ${ }^{1,2 *}$, Binqin Zhao ${ }^{1,2}$, Yiyu Lu ${ }^{1,2}$, Chengwei Liu ${ }^{1,2}$ and Chenpeng Song ${ }^{1,2}$ \\ ${ }^{1}$ State Key Laboratory of Coal Mine Disaster Dynamics and Control, Chongqing University, \\ Chongqing 400044 \\ ${ }^{2}$ National \& Local Joint Engineering Laboratory of Gas Drainage in Complex Coal Seam, \\ Chongqing University, Chongqing 400044
}

Email: xbwei33@cqu.edu.cn

\begin{abstract}
Considering that the methane drainage radius cannot speedily and accurately be confirmed after high pressure water jet slotting, a model for slotting the radius of high pressure water jet slotting is established based on the theory of round turbulent jet and Loland damage model. Combining the linear seepage theory and nonlinear seepage theory, this paper proposes the seepage model for various seepage statuses around the slot. The methane drainage radius after using a high pressure water jet consists of three parts: slotting radius, linear seepage area and nonlinear seepage area. Taking Zhongliangshan Mine as an example, the calculated results show that the slotting radius is $1.57 \mathrm{~m}$. The linear seepage areas range from $1.57 \mathrm{~m}$ to $4.81 \mathrm{~m}$, and the nonlinear seepage areas range from $4.81 \mathrm{~m}$ to $9.36 \mathrm{~m}$ from the center of borehole, respectively. Field experimentation shows that the radius of slotting reaches $1.5 \mathrm{~m}$. The effective drainage radius is $5 \mathrm{~m}$ and the radius of influence is $9 \mathrm{~m}$. It is relatively consistent with the theoretical calculations.
\end{abstract}

Keywords: Methane, High pressure water jet slotting, Methane flow field, Effective drainage radius.

\section{INTRODUCTION}

Gas pre-drainage through a borehole in coal seam is the primary means for disaster control of mine gas [1]. However, with the development of depth in underground coal mining, the characteristics of low permeability and high adsorption [2] of coal seam become more apparent, as well as high crustal stress and spontaneous combustion [3]. Recently, high-pressure water jet cutting technology [4-5] has been gradually applied and promoted in gas extraction. Shen Chunming, Lin Boquan et al. [4] have studied the impact of high-pressure water jet cutting on permeability of coal. Lu Yiyu et al. [6] have looked into the mechanism of improving gas permeability of coal seam based on the analysis of coal matrix shrinkage facilitated by water jet. As an important basis of boreholes location for gas pre-drainage, the extracting radius after high-pressure water jet cutting at present is mainly determined by on-site subjective experience. Oversized spacing of boreholes is likely to cause dead extraction zones, while an undersized one may result in increasing of hole numbers and air leakage and cause a loss to manpower and material. Therefore, it is vital for engineers to determine a method to determine the extracting radius of underground coal seam after high-pressure water jet cutting, so as to guide the underground water jet cutting drilling and establish a rational layout of gas drainage through a borehole.
Scholars globally have determined the extracting radius of coal-bed gas mainly according to gas pressure measurement, the coal-gas coupling model and tracer gas determination. Liu Sanjun et al. [7] in accordance with the relevant parabolic equation and gas re-drainage rate indicators, have proposed an extracting radius determination method on the basis of gas-bearing capacity, which proves to be more effective than pressure measurement. Yin Guangzhi et al. [8] taking into consideration the stress distribution after coal mine structure excavation and the impact of coal matrix's adsorption swelling on the coal, have theoretically established the coalgas coupling model and determined gas extracting radius. Xin Ming [9] researched the radius determining method by using SF6 as the tracer gas. There are disagreements over "gas predrainage rate of $30 \%$ " and "gas pressure of lower than 0.74MPa", [9] and it is difficult to apply the coal-gas coupling model to an actual site due to the complex coupling process and numerous parameters involved. Moreover, the tracer gas measuring is difficult to operate on site, and the test results cannot be widely applied. Therefore, this paper uses the theory of round turbulent jet and Loland damage model to study the depth-affecting factors of a high-pressure water jet cutting, and analyzes the influences of varied flow regimes of gas around the coal seam according to the linear flow and low-speed non-linear flow theory, so as to establish the radius model of gas around the coal seam with varied flow regimes. Finally, the gas extracting radius model after high-pressure 
water jet cutting is established, and relevant theoretical calculation results are verified based on field experimentation.

\section{HIGH-PRESSURE WATER JET CUTTING RADIUS}

The high-pressure water jet cutting is a typical round turbulent jet. In accordance with the axial velocity reduction law of round turbulent jets [10]. The momentum flux of each section conserves and equals the momentum flux of the nozzle.

$$
J=\int_{0}^{\infty} \rho u^{2} \cdot 2 \pi r d r=\rho u_{0}^{2} \pi r_{0}^{2}
$$

where, $J$ is momentum flux, $\rho$ is density of water, $u$ is jet axial velocity, $r$ is jet section radius, $u_{0}$ is nozzle exist flow velocity, $r_{0}$ is nozzle radius.

Since there are similarities in the sectional velocity distribution of the main sector of the jet, the jet axial velocity $u$ and jet core velocity $u_{m}$ present the Gaussian distribution.

$u=u_{m} \exp \left(-\frac{r^{2}}{b^{2}}\right)$

where, $b$ is the yet width. Taking $b_{0}$ as the half-thickness, when $y=b_{0}, u=u_{m} / e$; substitute $u=u_{m} / e$ into formula (1) for integration, and:

$$
\begin{aligned}
& \int_{0}^{\infty} u^{2} \cdot 2 \pi r d r=2 \pi u_{m}^{2} \int_{0}^{\infty} \exp ^{2}\left(\frac{r^{2}}{b_{0}^{2}}\right) r d r \\
& =2 \pi u_{m}{ }^{2} \cdot \frac{b_{0}^{2}}{4} \int_{0}^{\infty} \exp \left(-\frac{2 r^{2}}{b_{0}^{2}}\right) d\left(\frac{2 r^{2}}{b_{0}^{2}}\right) \\
& =u_{0}{ }^{2} \frac{\pi d^{2}}{4}
\end{aligned}
$$

Set the jet thickness linear scalability to $b_{0}=\varepsilon x$, and substitute it into formula (3):

$$
\frac{u_{m}}{u_{0}}=\frac{1}{\sqrt{2} \varepsilon}\left(\frac{d}{x}\right)
$$

By experiment, Albertson [11] determined that $\varepsilon=0.114$, and formula (4) thus can be reformed into:

$u_{m}=\frac{6.2 u_{0} d}{x}$

It can be determined from formula (5) that, with the increase of standoff, the core velocity of the round turbulent jet gradually reduces.

The similarity of sectional velocity distribution reveals that the velocity distribution, after being processed by $u_{m}$ of the $x / H$ section and non-dimensionalized by half-width $b_{u}$, can be expressed as:

$$
\frac{u}{u_{m}}=\exp \left(-0.639 \eta_{u}^{2}\right)
$$

In the formula, $\eta_{u}=r / b_{u}$, and $r$ is the jet radial radius. It can be concluded that $b_{u} / x=0.1696$ according to literature [12]. Formula (5) is substituted into (6) to determine the velocity distribution of the jet section:

$$
u=6.2 \exp \left(-0.639 \eta_{u}{ }^{2}\right) d \frac{u_{0}}{x}
$$

Assume that the jet blowing direction is perpendicular to the coal in the cutting, ignore the variation of jet flow and take the velocity as 0 when the flow is completed. When radial distance equals $r$, according to the momentum theorem, the jet acting force on the coal can be concluded as $F_{r}$ :

$$
F_{r}=-\rho\left[\pi(r+d r)^{2}-\pi r^{2}\right] u=-4 \rho u \pi d r
$$

Bradshaw et al. [13] believe that, under the jet action, damage to the coal occurs within the jet impact region. The research of Beltaos et al. [14] indicates that the round turbulent jet impact region radius is $\mathrm{r} \approx 0.22 \mathrm{H}$, and by integrating $F_{r}$ within the jet impact region it can be conducted that:

$F=24.8 \rho \pi d u_{0}\left(0.02 H^{3}-0.02 H^{3} e^{-\frac{4.884}{H}}-0.009 H^{2} e^{-\frac{4.884}{H}}\right)$

Therefore, the stress of the unit area of the coal produced by jet impact is:

$F=24.8 \rho \pi d u_{0}\left(0.02 H^{3}-0.02 H^{3} e^{-\frac{4.884}{H}}-0.009 H^{2} e^{-\frac{4.884}{H}}\right)(10)$

The Lonland mathematic model is used for expressing the damage to the coal. The Lonland model agrees that, before the peak stress $\left(\varepsilon \leq \varepsilon_{\mathrm{p}}\right)$ is reached, coal structural cracks grow and expand only within the volume element and remain within a low limitation [15]. The damage evolution equation this time is:

$\left\{\begin{array}{c}\tilde{\sigma}=E \varepsilon /\left(1-D_{0}\right) \\ \sigma=\tilde{\sigma}(1-D) \\ D=D_{0}+C_{1} \varepsilon^{\beta}\end{array}\right.$

After the peak stress $\left(\varepsilon_{\mathrm{p}} \leq \varepsilon \leq \varepsilon_{\mathrm{u}}\right)$ is reached, cracks expand unsteadily within the failure zone, and the damage evolution equation for this period is:

$\left\{\begin{array}{c}\lim _{x \rightarrow \infty} \tilde{\sigma}=E \varepsilon_{p} \\ \sigma=\tilde{\sigma}(1-D) \\ D=D\left(\varepsilon_{p}\right)+C_{2}\left(\varepsilon-\varepsilon_{p}\right)\end{array}\right.$

In the formula, $\quad \beta=\sigma_{\mathrm{p}} /\left(E \varepsilon_{p}-\sigma_{p}\right)$, $\mathrm{C}_{1}=\varepsilon_{p}\left(1-D_{0}\right) /(1+\beta), \mathrm{C}_{2}=\left[1-D\left(\varepsilon_{p}\right)\right] / \varepsilon_{\mathrm{u}} \varepsilon_{\mathrm{p}} ; \varepsilon$ is the tensile strain, $\varepsilon_{u}$ is the ultimate strain, $\varepsilon_{p}$ is the strain value of peak stress, $\sigma_{p}$ is the peak stress of the coal, $\tilde{\sigma}$ is the effective stress when considering the damage effect, $E$ is the elasticity modulus of the coal, $D_{0}$ is the initial damage, and $D\left(\varepsilon_{p}\right)$ is the damage when the peak stress is reached.

It can be conducted from formulas (11) and (12) that the stress acting on the coal in a varying strain stage is: 
$\left\{\begin{array}{c}\sigma=\tilde{\sigma}\left(1-D_{0}-C_{1} \varepsilon^{\beta}\right) \quad\left(\varepsilon \leq \varepsilon_{P}\right) \\ \sigma=\tilde{\sigma}\left\{1-\left[D_{0}+C_{1} \varepsilon_{P}{ }^{\beta}+C_{2}\left(\varepsilon-\varepsilon_{P}\right)\right]\right\} \\ \left(\varepsilon_{P}<\varepsilon \leq \varepsilon_{u}\right)\end{array}\right.$

Assuming that the coal is uniaxial strained when it is impacted by the high-pressure water jet, it will be damaged when shear force acting on it exceeds the shearing strength according to the Mohr-Coulomb Criterion:

$|\tau|=c+\sigma \tan \varphi$

By substituting formula (13) into (14), the effective stress value $\tilde{\sigma}$ when the coal is damaged can be acquired as:

$$
\tilde{\sigma}=\frac{\tau-c}{\tan \varphi\left\{1-\left[D_{0}+C_{1} \varepsilon_{p}{ }^{\beta}+C_{2}\left(\varepsilon-\varepsilon_{p}\right)\right]\right\}}
$$

Combining formula (15) and (12) can acquire the critical stress when the coal is damaged:

$$
\sigma=\frac{(\tau-c)\left(1-D_{0}\right)}{\tan \varphi\left\{1-\left[D_{0}+C_{1} \varepsilon_{p}{ }^{\beta}+C_{2}\left(\varepsilon-\varepsilon_{p}\right)\right]\right\}}
$$

With an increase in standoff, the core velocity and impact force of the turbulent jet reduces exponentially. When the standoff is $\mathrm{H}=\mathrm{R}$, the jet impact force equals the critical value when the coal is damaged. In this case, $\mathrm{H}$ is the seam radius cut by the jet.

$$
\begin{aligned}
& \frac{F}{\pi(0.22 H)^{2}}=22904.28 \rho d \sqrt{P} \times \\
& \left(0.02 H-0.02 H e^{-\frac{4.884}{H}}-0.099 e^{-\frac{4.884}{H}}\right) \\
& =\frac{(\tau-c)\left(1-D_{0}\right)}{\tan \varphi\left\{1-\left[D_{0}+C_{1} \varepsilon_{p}{ }^{\beta}+C_{2}\left(\varepsilon-\varepsilon_{p}\right)\right]\right\}}
\end{aligned}
$$

where $P$ is the pumping pressure. In the formula, parameters related to the coal, such as $c, \tau, \varphi, \varepsilon_{p}$ and $E$, can be directly tested for hard coal. Since it is difficult to do sample preparation and test for soft coal and fragmentized coal, this paper follows Hoek-Brown's experience to determine the value of $c$ and $\varphi[16,17] . \sigma_{p}$ (in MPa) is about ten times of $f$, the firmness coefficient of the coal, and the value of $f$ can be measured in conformance with the national standard [18]. The soft coal has poor strength of extension, with a stretching strain lower than $10^{-4}$ in most cases, so the coal's tensile strain $\varepsilon$ can be ignored [19]. The uniaxial compressive strength, elasticity modulus $E$ and peak strain $\varepsilon_{p}$ can be calculated via the formula below [20]. Porosity can be measured with the Poremaster 33 high pressure porosity apparatus, and it can be ascertained that porosity is the coal's initial damage $\mathrm{D}_{0}$.

$E=0.4029 R_{0}+3.4724$

$\varepsilon_{p}=\frac{\sigma_{p}}{E}$

In the formula, $R_{0}$ is the reflectance of vitrinite in the coal.

\section{GAS SEEPAGE FIELD AROUND THE SEAM}

When gas flows around the seam, the gas flow areas can be divided into linear seepage zone, low-speed non-linear seepage zone and diffusion zone according to the gas flow status. It is further assumed that the gas transport within the diffusion zone will not affect the extracting [21].

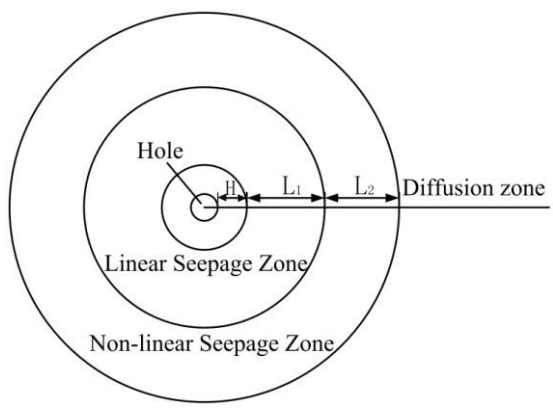

Figure 1. Field area around the slot

\subsection{Linear seepage zone}

It can be concluded according to Darcy law that [22]:

$v=10^{-3} \frac{K \Delta P}{\mu L_{1}}$

In the formula, $v$ is the seepage velocity, $\mathrm{m} / \mathrm{s} ; K$ is the permeability $\mu \mathrm{m}^{2} ; \mu$ is the gas dynamic viscosity $\mathrm{mPa} \cdot \mathrm{s} ; \Delta P$ is the pressure difference $\mathrm{MPa} ; L_{l}$ is the gas migration distance $m$.

Kajahoff [23] presented an expression of Reynolds number that is generally accepted as rational, and it is believed that $\mathrm{Re}=10^{-4}$ is the boundary of linear seepage and non-linear seepage;

$\operatorname{Re}=10^{-4} \frac{v \sqrt{K} \rho}{17.50 \mu \phi^{3 / 2}}$

In the formula, $\rho$ is the density of the fluid, $\mathrm{g}_{\mathrm{cm}} \mathrm{cm}^{3} ; \phi$ is the porosity. By substituting formula (20) into (21) the radius $L_{1}$ of the linear seepage zone can be acquired:

$L_{1}=\frac{10^{-15} \rho \Delta p k^{\frac{3}{2}}}{17.50 u^{2} \phi^{\frac{3}{2}} \operatorname{Re}}$

\subsection{Low-speed non-linear seepage zone}

When gas flows in the coal seam with low air permeability, since the coal pore porosity is less than the mean free-path of gaseous molecule, the gaseous molecule will collide against the pore wall. The impact brought about by such a collision on seepage law, at the macro level, manifests as the slippage effect [23]. Wu Fan et al. [24] find that the slippage effect is conditional. Gas start-up pressure gradient $\left(\lambda_{B}\right)$ exists when the velocity is lower. When the pressure gradient exceeds the start-up pressure gradient, gas movement within the coal seam is mainly low-speed non-linear seepage; when the pressure gradient is lower than the start-up pressure gradient, 
gas movement is only in dispersal mode. According to the low-speed non-linear seepage law [25], it can be drawn that:

$V= \begin{cases}\frac{\mathrm{K}}{\mu}\left(1+\frac{2 b}{P_{1}+P_{2}}\right) \frac{\Delta P}{L}, \frac{\Delta P}{L}>\lambda_{B} \mathrm{~b} \\ 0, \frac{\Delta P}{L} \leq \lambda_{B}\end{cases}$

In the formula, $P_{1}$ and $P_{2}$ represent the gas pressure of the inflow and outflow ends respectively; $b$ is the Klinkenberg constant. Meanwhile, Guo Hongyu et al. [26] researched the relationship between the gas start-up pressure gradient and coal permeability though experiments.

$$
\lambda_{B=0.0113 K^{-0.33034}}
$$

Within a low-speed non-linear seepage zone, when $\mathrm{v}=0$ in formula (21), $L_{2}$ is the maximum distance of gas movement within the non-linear seepage zone, thus:

$$
L_{2}=\Delta p / \lambda
$$

The gas extracting radius can be calculated according to formulas (22) and (25) as $R=H+L_{1}+L_{2}$

\section{FIELD APPLICATION}

The experiment was performed at the Zhongliangshan mine. The gas extracting radius of the coal seam after highpressure water jet cutting has been reviewed. The borehole location is shown in Figure 2. The parameters of the coal seam are shown in Table 1.

Table 1. Parameters of the coal seam

\begin{tabular}{llll}
\hline Parameter & Value & Parameter & Value \\
\hline $\begin{array}{l}\text { Methane } \\
\text { Density }\left(\mathrm{kg} / \mathrm{m}^{3}\right)\end{array}$ & 0.717 & Porosity & 0.08 \\
$\begin{array}{l}\text { Methane } \\
\text { Pressure }(\mathrm{MPa})\end{array}$ & 2.2 & Permeability $\left(\mathrm{um}^{2}\right)$ & $\begin{array}{l}1.5 \times 10^{-} \\
5\end{array}$ \\
$\begin{array}{l}\text { Barometric } \\
\text { Pressure }(\mathrm{MPa})\end{array}$ & 0.1 & $f$ Value & 0.3 \\
$\begin{array}{l}\text { Negative } \\
\text { Pressure }(\mathrm{kPa})\end{array}$ & 35 & Cohension$(\mathrm{MPa})$ & 0.84 \\
$\begin{array}{l}\text { Viscosity }(\mathrm{mPa} \cdot \mathrm{s}) \\
\text { Vitrinite Reflectance }\end{array}$ & $1.08 \times 10^{-2}$ & Fraction Angle $\left(^{\circ}\right)$ & $56^{\circ}$ \\
\hline
\end{tabular}

The slotting radius of the coal seam and the linear seepage can be calculated by the above parameters and formula as $\mathrm{H}=1.57 \mathrm{~m}$, and $\mathrm{L}_{1}=3.24 \mathrm{~m}$, respectively. The start-up pressure gradient of the coal seam can be calculated by formula (24) as $\lambda_{\mathrm{B}}=0.425 \mathrm{MPa} / \mathrm{m}$. It can be calculated by formula (25) that, in non-linear zone, $\mathrm{L}_{2}=4.55 \mathrm{~m}$.

Based on the above analysis, when the extracting subpressure is $35 \mathrm{kPa}$, the area $4.81 \mathrm{~m}$ away from the drill center is linear area; and the low-speed non-linear and diffusion area are $4.81 \mathrm{~m} \sim 9.36 \mathrm{~m}$ and $9.36 \mathrm{~m}$ away from the drill center respectively.

According to the Regulation on Coal and Gas Outburst Prevention, this paper takes a gas pressure of lower than $0.74 \mathrm{MPa}$ as the review index. Based on the surrounding rock conditions of the roadway and gas geological conditions of the Zhongliangshan Mine, this paper arranges the gas pressure inspection holes on the \#17 drill site and along its path. This paper set a hole for high pressure slotting (\#5) and eight inspection holes (\#1, \#2, \#3, \#4, \#6, \#7, \#8 and \#9); Note that \#6 hole is taken as the inspection hole of both the pressure and slotting radius. The layout of the slotting and inspection holes is shown in Figure 2.

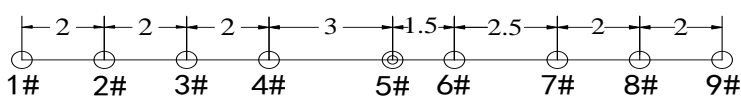

Figure 2. Diagrammatic sketch of boreholes (m)

The pressure measurement and drilling are performed according to relevant criterion [27]. For the drilling process, firstly, eight inspection holes (\#1, \#2, \#3, \#4, \#6, \#7, \#8 and \#9) were drilled, then the holes were immediately sealed. The slotting hole (\#5) would not be constructed until the gas pressure became stable, which turned out to be the $64^{\text {th }}$ day. There was water leakage from \#6 during the high pressure water jet slotting, which indicated the slotting radius of $1.5 \mathrm{~m}$. Gas pressure changes of all inspection holes after the slot was cut are shown in Figures 3 and 4.

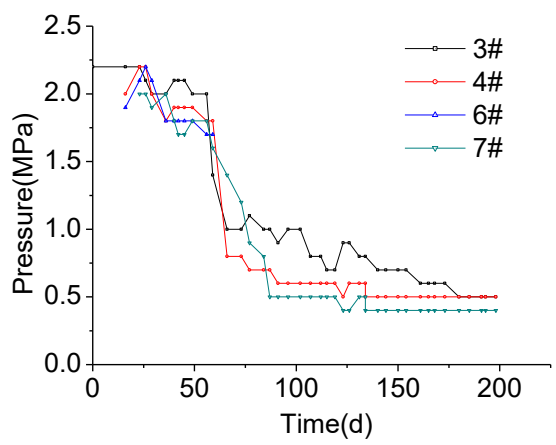

Figure 3. Pressure curve graph of \#3, \#4, \#6 and \#7

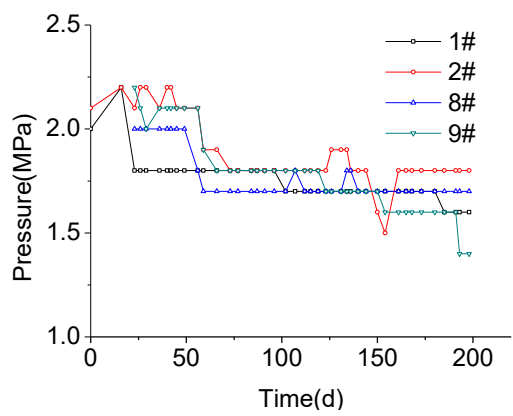

Figure 4. Pressure curve graph of \#1, \#2, \#8 and \#9

The variation of gas pressure curves indicates that when the hole (\#5) was cut, the gas pressure of the adjacent inspection holes \#4, \#6, \#7 decreased sharply and fell to below $0.74 \mathrm{MPa}$, which indicates that the natural gaseous emission radius can reach $4 \mathrm{~m}$ before the gas drainage and after the seam cutting. The gas pressure of \#3, 5m away from the slotted hole, fell to below 0.74MPaafter after 51 days of drainage. The gas pressure in areas within $5 \mathrm{~m}$ away from the seam hole reached the standard, and gas pressure within $9 \mathrm{~m}$ away decreased by more than $10 \%$. This suggests that the effective gas extracting radius reached $5 \mathrm{~m}$ after the seam cutting and the radius of influence reached $9 \mathrm{~m}$, which is consistent with the theoretical calculation results. 
Within the margin of engineering error, this method can be used for calculating the gas extracting radius after highpressure water jet cutting for various coal seams. In addition, it has provided a theoretical basis for the reasonable design of pre-drainage drilling.

\section{CONCLUSIONS}

According to this paper, some hightlights are:

(1) Combined with Loland damage model, this paper establishes a radius model of high-pressure water jet cutting based on the turbulent jet velocity's change rule with the distance variation. The experience parameters of the coal are adopted to calculate the radius $\mathrm{H}$ of the seam of the coal seam in the Zhongliangshan Mine, and $\mathrm{H}=1.57 \mathrm{~m}$.

(2) This paper establishes a radius model of gas around the coal seam with varying flow regimes based on the linear flow and low-speed non-linear flow theory. According to the parameters of the coal seam in the Zhongliangshan Mine, the calculation result shows that areas $1.57 \sim 4.81 \mathrm{~m}$ away from the drill center are linear seepage zones, and those $4.81 \sim 9.36 \mathrm{~m}$ away from the drill center are low-speed non-linear seepage zones.

(3) The on-site experimental research of the coal seam in the Zhongliangshan Mine suggests that when the seam radius reaches $1.5 \mathrm{~m}$, areas within a $4 \mathrm{~m}$ radius around the seam are up to the standard even without any gas extraction; after 51 days of gas extraction, gas pressure within a $5 \mathrm{~m}$ radius around the seam reaches the standard; and after 131 days of gas extraction, the change in gas pressure within a $9 \mathrm{~m}$ radius around the seam exceeds $10 \%$.

(4) Since the comprehensive parameter testing method is still lacking for soft and fragmentized coal, we still need practical experience in parameter selection, which is a major cause of errors.

\section{ACKNOWLEDGMENT}

This work was supported by National Natural Science Foundation of China (No. 51104191); (No. 51374258) and Major State Basic Research Development Program (No. 2014CB239206).

\section{REFERENCES}

[1] Yu Qixiang, Theory and Technology of Coal Mine Disaster Control, Xuzhou, Choina: China University of Mining and Technology Press, 2012,ch.7, pp.119-121.

[2] Wang Haiyan, Cheng Yafei and Yu Bo, "Adsorption effect of overlaying strata on carbon dioxide in coalfield fire area." Int $J$ of Heat and Tech, vol. 33, no. 3, pp. 11-18, 2015. DOI: 10.18280/ijht.330302.

[3] Zhang Hongfen and Gao Erxin, "3D simulation and influencing factors of loose top coal spontaneous combustion in roadway," Int J of Heat and Tech, vol. 33, no. 3, pp. 91-96, 2015. DOI: 10.18280/ijht.330313.

[4] Shen Chunming, Lin Baiquan and Wu Haijin, "Highpressure water jet slotting and influence on permeability of coal seams." J of China Coal Society, vol. 36, no. 12, pp. 2058-2063, 2011.

[5] Lu Yiyu, Ge Zhaolong, Li Xiaohong, et al., "Investigation of a self-excited pulsed water jet for rock cross-cutting to uncover coal," $J$ of China Uni of Mining \& Tech, vol. 39, no. 1, pp. 55-58, 2010.

[6] Lu Yiyu, Song Chenpeng, Liu Yong, et al., "Mechanism analysis of permeability based on promoting coal matrix shrinkage by water jet," $J$ of Chongqing Uni, vol. 34, no. 4, pp. 20-23, 2011.

[7] Liu Sanjun, Ma Gen, Lu Jie, et al., "Relative pressure determination technology for effective radius found on gas content," J of China Coal Society, vol. 36, no. 10, pp. 1715-1719, 2011.

[8] Yin Guangzhi, Li Minghui, Li Shengzhou, et al., “3D numerical simulation of gas drainage from boreholes based on solid-gas coupling model of coal containing gas." J of China Coal Society, vol. 38, no. 4, pp. 535541, 2013.

[9] Xin Ming, "Determination of gas extraction radius in coal seams by tracer technique with SF6." J of Anhui Uni of Sci and Tech, vol. 32, no. 1, pp. 64-66, 2012.

[10] Dong Zhiyong, Jetting Mechanics, Beijing, China: Science Press, 2006.

[11] Albertson M. L., Dai Y. B. and Bouwer H., "Diffusion of submerged jet." Transactions ASCE, vol. 115, pp. 639-664, 1950.

[12] Li Xiaohong, Lu Yiyu and Xiang Wenying, High Pressure Water Jet Theory and Application on Mining Engineerin. Chongqing, China: Chongqing University Press, 2007, ch. 2, pp. 45-53.

[13] Bradshaw P. and Love E. M., "The normal impingement of a circular jet on a flat plane," Great Britain Aeronautical Research Council, ARC Report Council, London, 1959.

[14] Beltaos S. and Rajartam N, "Plane turbulent impinging jet." Hydraulic Research, vol. 11, no. 1, pp. 29-59, 1973.

[15] K. E. Loland, "Continuous damage model for loadresponse estimation of concrete," Cement and Concrete Research, vol. 10, no. 3, pp. 395-402, 1980.

[16] Zhang Lang, "A new prediction index of coal and gas outburst," J of Mining \& Safety Engineering, vol. 30, no. 4, pp. 616-620, 2013.

[17] Huang Xiaohu, Wang Changming, Wang Tianzuo, et al., "Quantification of geological strength index based on discontinuity density of rock masses," Int J of Heat and Tech, vol. 33, no. 4, pp. 255-261, 2015. DOI: 10.18280/ijht.330434.34.

[18] Methods for determining coal hardness coefficient, GB/T 23561.12-2010, 2010.

[19] Qi Qingxin, Mao Debing, Fan Shaogang, et al., “A study of elasto-brittle-plasticity for coal in uniaxial tension test," the $7^{\text {th }}$ Academic Conference for Chinese Society for Rock Mechanics \& Engineering, Xi'an, 2002, pp. 181- 185 .

[20] Jienan Pan, Zhaoping Meng, Quanlin Hou, et al., "Coal strength and Young's modulus related to coal rank, compressional velocity and maceral composition," J of Structural Geo, vol. 54, pp. 129-135, 2013.

[21] Zhou Shining and Lin Baiquan, The Theory of Methane Occurrence and Percolation. Beijing, China: China Coal Industry Publishing House.1999.

[22] Li Xiaoping, Permeation Fluid Mechanics of Petrol and Gas, Beijing, China: Petroleum Industry Press, 2008.

[23] КатяХоув. Basis of Reservoir Physical. Beijing, China: Petroleum Industry Press, 1958.

[24] Wu Fan and Sun Lijuan, "A research on gas flow property and starting pressure phenomenon," Natural 
Gas Industry, vol. 21, no. 1, pp. 82-84, 2001.

[25] Feng Wenguang. Basic Porous Flow of Petrol and Gas. Beijing, China: Science Press, 2007, ch.2, pp.49-54.

[26] Guo Hongyu and Su Xianbo, "An experimental measurement of the threshold pressure gradient of coal reservoirs and its significance," Natural Gas Industry, vol. 30, no. 6, pp. 52-54, 2010.

[27] Method for Directly Determining the Pressure of Coal Seam Methane, AQ/T 1047-2007, 2007. 